\title{
Simulation and Experimental Study of the Three-phase Five-legged Transformer under DC Bias
}

\author{
Dong Xia \\ School of Electrical Engineering and Automation \\ Qilu University of Technology \\ Jinan, China \\ dongxia6078@163.com \\ * Corresponding Author
}

\begin{abstract}
This paper builds a novel coupled model which includes the electric circuit and the magnetic circuit of the three-phase five-legged transformer under direct current (DC) bias. In the magnetic circuit model, the eddy-currents, the iron core topology and the saturation characteristics of the core material are taken into account. The magnetomotive forces due to the eddy currents are given in the magnetic circuit equations. Combining the electrical circuit with the magnetic circuit, and considering the nonlinear characteristic curve of iron core, the nonlinear equations can be obtained. A series of mathematics transformation is performed and the numerical nonlinear algebraic equations are solved. Then the experimental work of the three-phase five-legged transformer under DC bias has been done. Finally, the simulation results and the experiment results are compared. They are in good agreement. Meanwhile, DC bias problem on the three-phase five-legged transformers is analyzed. It demonstrates that the validity of the simulation model and the accuracy of analyzing DC bias phenomenon of the three-phase five-legged transformer.
\end{abstract}

Keywords-three-phase five-legged transformer; DC bias; eddy-current; saturation characteristic; nonlinear

\section{INTRODUCTION}

DC bias phenomenon is an abnormal work station of transformers. There are two reasons which arise DC bias. One is the monopolar ground circuit operation mode or the bipolar asymmetrically operation mode of the HVDC transmission system[1-2]. In this case, DC will pass by the windings of the transformers through the earthed neutral point. The other is Geomagnetically Induced Current (GIC) which is caused by the interaction with the geomagnetic field and the dynamic movement of the ionic wind[3]. The change of the geomagnetic field produces an electric potential gradient, which gives rise to low-frequency induction current. It is DC because its frequency is very low. The work point of the transformer under DC bias will shift, and its iron core material will be saturated which affects the normal work of the transformer seriously. This produces lots of bad influence, for example, the distortion of the exciting current; the heavy noise; the serious vibration; the increase of transformer's leakage and losses; the local heating of metal structures; the malfunction of relay protection devices of electric power system, et al. So DC bias phenomenon of the transformer is concerned.
Some simulations and experiments have been done by a lot of researchers. The main research contents included four aspects as follows: (1) experimental research of the vibration, great noise, local high temperature of the transformer[4-6]; (2) harmonics analysis of the exciting current[7-9]; (3) measurement of magnetization characteristic of ferromagnetic material[10]; (4) research of restrain measures of DC bias[11-12]. Some achievements and progress have been obtained at above mentioned aspects.

The eddy currents, the iron core topology and the saturation characteristic of the three-phase five-legged transformer under DC bias have to be considered sufficiently. This paper builds a novel coupled model which includes the electric circuit and the magnetic circuit considering the above factors. The magnetomotive forces due to the eddy currents are included in magnetic circuit equations. Coupled these equations with the electrical circuit equations, the nonlinear equations are obtained. The analysis of the three-phase five-legged transformer under DC bias can be accomplished through solving the equations.

\section{THE COUPLED MODEL OF TRANSFORMER}

\section{A. The Model of the Electic Circuit}

The equivalent electric circuit of the no-load threephase five-legged transformer is shown as Fig.1. Where $u_{1}, u_{2}$ and $u_{3}$ are the three-phase AC voltages. $R_{1 \mathrm{a}}, R_{2 \mathrm{~b}}, R_{3 \mathrm{c}}$, $R_{1 \mathrm{~A}}, R_{2 \mathrm{~B}}, R_{3 \mathrm{C}}$ are the resistances of the primary and secondary windings respectively. $e_{1 \mathrm{a}}, e_{2 \mathrm{~b}}, e_{3 \mathrm{c}}, e_{1 \mathrm{~A}}, e_{2 \mathrm{~B}}, e_{3 \mathrm{C}}$ are the induced electromotive forces of the primary and secondary windings respectively. $i_{1}, i_{2}$ and $i_{3}$ are currents of the primary windings. $L_{1 \mathrm{a}}, L_{2 \mathrm{~b}}$ and $L_{3 \mathrm{c}}$ are the equivalent leakage magnetic inductions which couple with the primary windings. $L_{1 \mathrm{~A}}, L_{2 \mathrm{~B}}$ and $L_{3 \mathrm{C}}$ are the equivalent leakage magnetic inductions which couple with the secondary windings. $U_{0}$ is the DC voltage.

The equations can be derived as (1).

$u_{j}+U_{0}-R_{j p} i_{j}-L_{j p} \frac{d i_{j}}{d t}=-e_{j p}=N \frac{d \phi_{j}}{d t}, j=1,2,3$

Where small letter subscripts $p$ represents the primary side parameters. $\Phi_{\mathrm{j}}$ is the iron core legs magnetic flux of the transformer. 


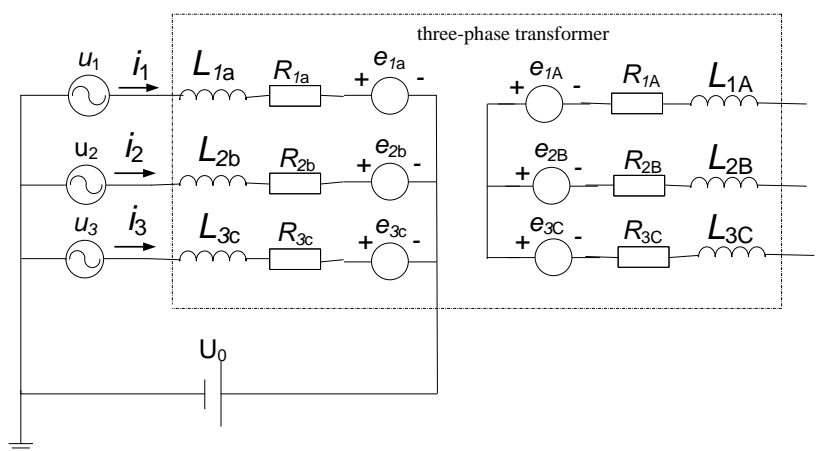

Figure 1. Equivalent electric circuit of the three-phase five-legged transformer under DC bias

\section{B. The Model of the Magnetic circuit}

The equivalent magnetic circuit of the three-phase five-legged transformer is shown as Fig.2. Where $F_{\mathrm{a}}, F_{\mathrm{b}}$ and $F_{\mathrm{c}}$ are the magnetomotive forces produced by the primary winding current. $R_{\mathrm{k}}$ and $\Phi_{\mathrm{k}}(\mathrm{k}=1 \sim 7)$ are the reluctances and magnetic fluxes of the iron core legs, yokes and side legs. $R_{8}$ and $\Phi_{8}$ are the leakage reluctance and flux. $F_{\mathrm{ek}}(\mathrm{k}=1 \sim 7)$ are the magnetomotive forces produced by eddy currents.

In the magnetic circuit model, the eddy currents are considered. The magnetomotive forces produced by eddy currents are given by[13]

$$
\begin{gathered}
F_{e}=k_{e} \frac{d \phi}{d t} \\
k_{e}=\frac{\sigma d^{2} l}{12 A}+\left(\frac{G d \tau H_{o} \sigma l^{2}}{A}\right)^{\frac{1}{2}}\left(\frac{d \phi}{d t}\right)^{-\frac{1}{2}}
\end{gathered}
$$

Where $A$ and $l$ are the cross-section area and the length of the iron core legs respectively. $\sigma$ is the electrical conductivity. $d$ is the thickness of the laminations. $\tau$ is the width of the laminations. $G=0.1356 . H_{0}$ is a parameter with dimensions $\mathrm{A} / \mathrm{m}$ representing the internal potential experienced by domain walls in the magnetic laminations.

The nonlinear equation of the core material with the single value curve is given by:

$$
B=f(H)
$$

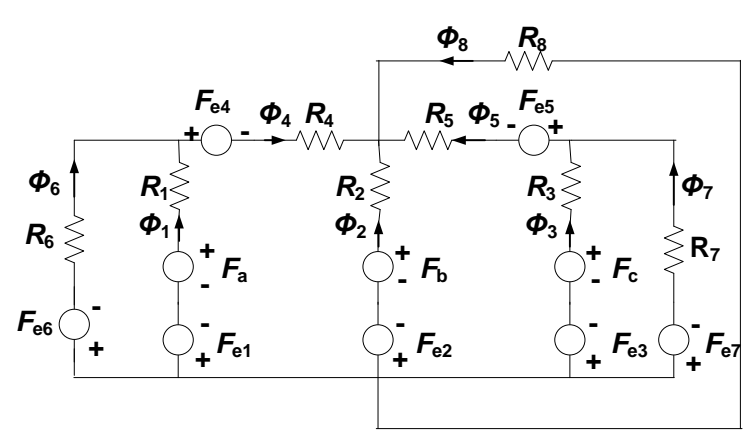

Figure 2. Equivalent magnetic circuit of the three-phase five-legged transformer under DC bias
The nonlinear reluctances of the magnetic circuit are given by

$$
R_{k}=\frac{l}{A \mu}=\frac{l}{A} \frac{H}{B}
$$

The magnetic circuit equations (6) can be obtained according to Fig.2.

$$
\left\{\begin{array}{l}
R_{1} \phi_{1}-R_{6} \phi_{6}=F_{a}+F_{e 6}-F_{e 1} \\
R_{1} \phi_{1}+R_{4} \phi_{4}-R_{2} \phi_{2}=F_{a}+F_{e 2}-F_{b}-F_{e 1}-F_{e 4} \\
R_{3} \phi_{3}+R_{5} \phi_{5}-R_{2} \phi_{2}=F_{c}+F_{e 2}-F_{b}-F_{e 3}-F_{e 5} \\
R_{3} \phi_{3}-R_{7} \phi_{7}=F_{c}+F_{e 7}-F_{e 3} \\
R_{2} \phi_{2}-R_{8} \phi_{8}=F_{b}-F_{e 2} \\
\phi_{1}+\phi_{6}=\phi_{4} \\
\phi_{3}+\phi_{7}=\phi_{5} \\
\phi_{2}+\phi_{4}+\phi_{5}+\phi_{8}=0
\end{array}\right.
$$

Substituting (2), (5) into (6) and carrying on transformation and reorganization, the matrix equation is

$$
R \phi_{j}=F(j=1,2,3)
$$

Finally, the nonlinear differential-algebraic equations can be obtained from the formula (1) and (7). Then they are solved iteratively by the Newton Raphson method. By selecting the appropriate time step, the stability and accuracy of the solution can be assured.

\section{SIMULATION RESULTS}

The main parameters of the double windings threephase five-legged transformer are listed as follows: rated capacity: $112.5 \mathrm{MVA}$, rated transformation ratio: $110 \mathrm{KV} /$ $16 \mathrm{KV}$, cross-section area of iron core and yoke: $0.4536 \mathrm{~m}^{2}$, length of iron core: $3.59 \mathrm{~m}$, length of yoke: $2.656 \mathrm{~m}$, cross-section area of side leg: $0.1814 \mathrm{~m}^{2}$, length of side leg: $7 \mathrm{~m}$
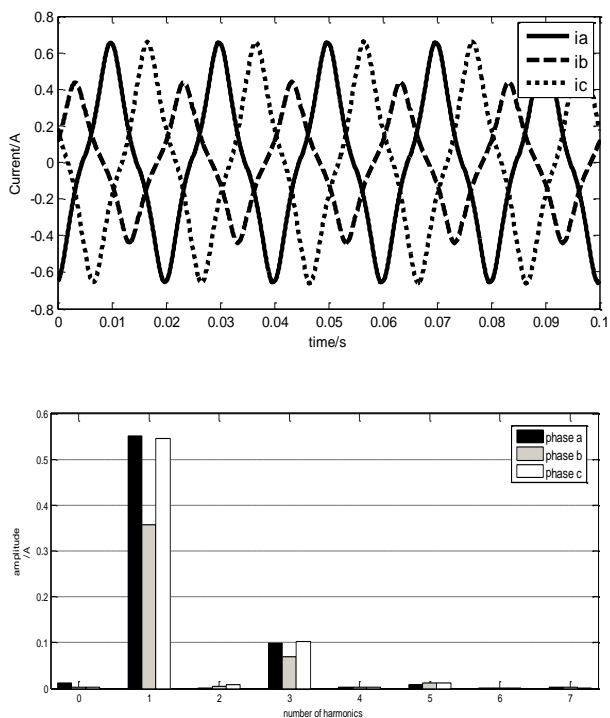

Figure 3. Exciting current and harmonic distribution without DC bias 


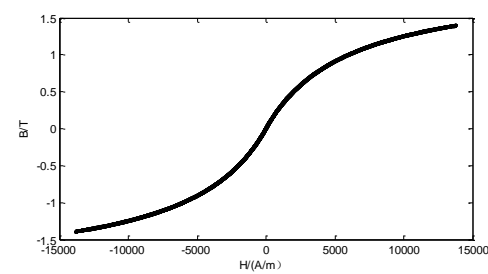

(a) phase a

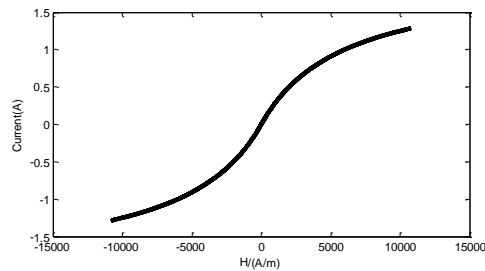

(b) phase b

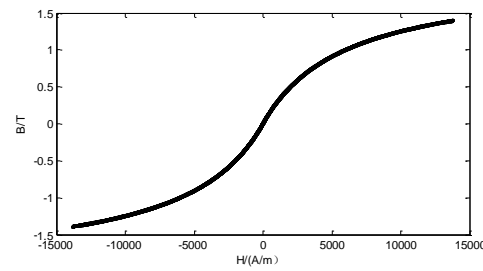

(c) phase c

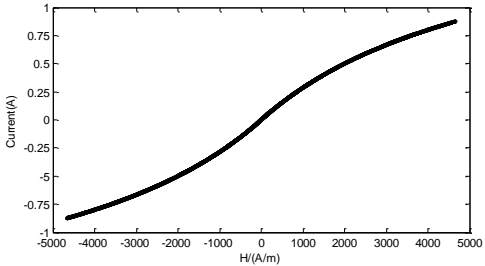

(d) side leg

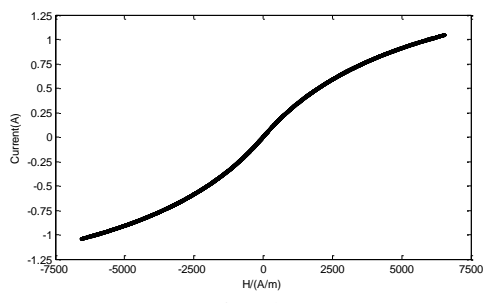

(f) yoke

Figure 4. Nonlinear characteristic curve without DC bias

The calculation results of the exciting currents, harmonic distributions and nonlinear curves without DC bias are shown as Fig.3 and Fig.4. It can be seen that the exciting currents are symmetrical at positive and negative $\mathrm{Y}$ axis and do not get distorted without DC bias. The first and third odd harmonics are the main ingredient of the exciting currents. The nonlinear characteristic curves do not shift.

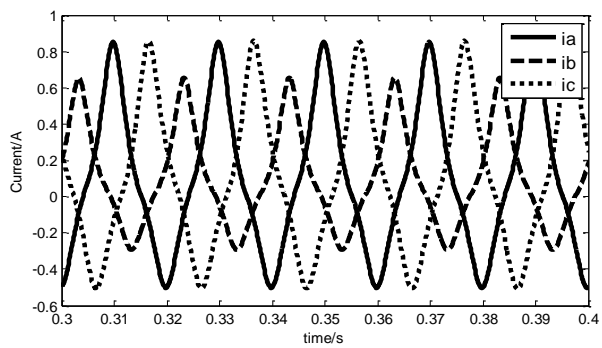

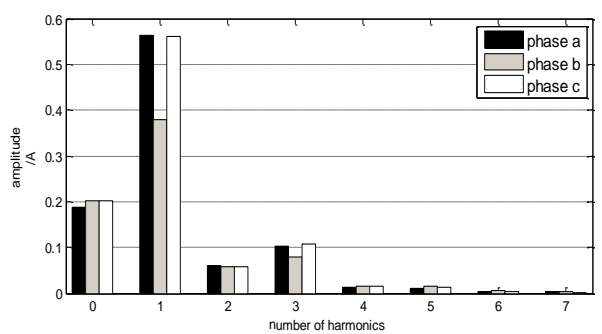

Figure 5. Exciting current and harmonic distribution with DC bias $\left(I_{D C}=0.593 \mathrm{~A}\right)$

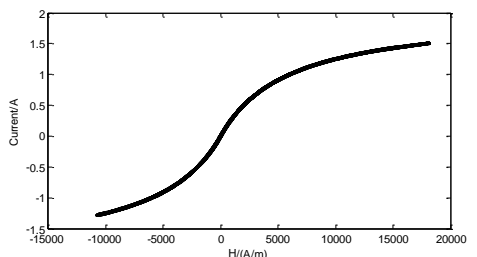

(a) phase a

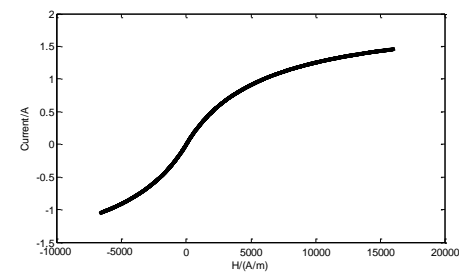

(b) phase $b$

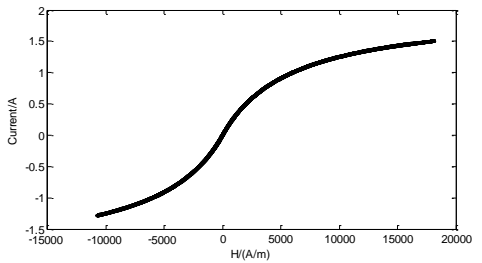

(c) phase c

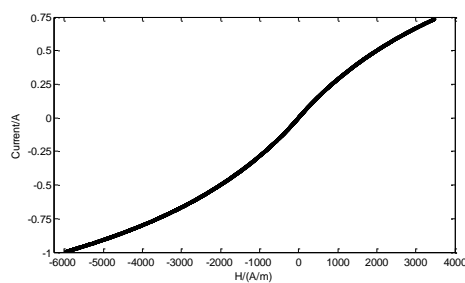

(d) side leg

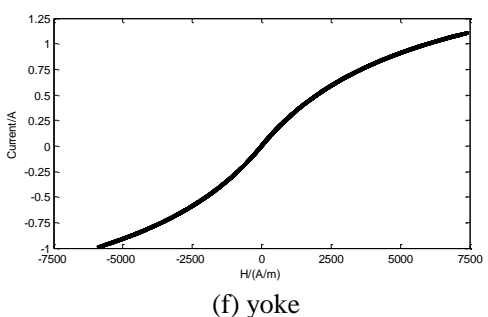

Figure 6. Nonlinear characteristic curve with DC bias $\left(I_{D C}=0.593 \mathrm{~A}\right)$

The calculation results of the exciting currents, harmonic distributions and nonlinear curves with DC bias are shown as Fig.5 and Fig.6. It can be seen that the exciting currents are not symmetrical at positive and negative $\mathrm{Y}$ axis any more and get distorted with DC bias. The even harmonics such as the secondary and fourth harmonics occurred according to the harmonics analysis. The nonlinear characteristic curves of the phase $a, b$ and $c$ tend to saturate at the positive half of the $\mathrm{Y}$ axis, and those of the side legs and yokes shift up without reaching 
saturation. The distortion of the exciting currents, the saturation and deviation of the nonlinear characteristic curves will become more serious with the increase of DC current.

\section{EXPERIMENTAL STUDY}

Fig.7 is the measurement circuit of the three-phase five-legged transformer under DC bias. Where $u_{1}, u_{2}$ and $u_{3}$ are the three-phase AC voltages. $R_{\mathrm{e}}$ is the internal resistance. $U_{0}$ is the DC power supply. $R$ is the variable resistance. The capacity of the transformer is 200VA, and the ratio is $380 \mathrm{~V} / 38 \mathrm{~V}$.

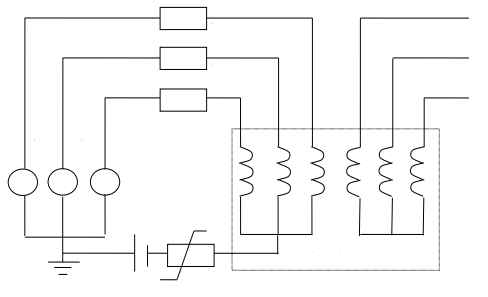

transformer

Figure 7. The measurement circuit of the transformer

Fig.8 and Fig.9 are the comparative graphics of the simulation results and the measurement results. It can be seen that the waveforms are in good agreement.

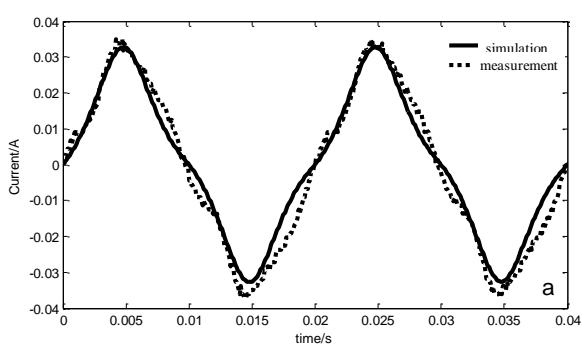

(a) a phase

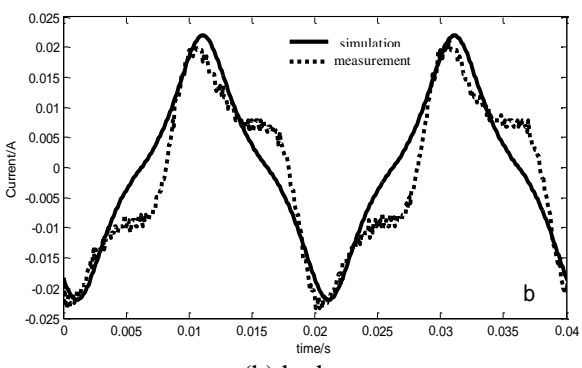

(b) b phase

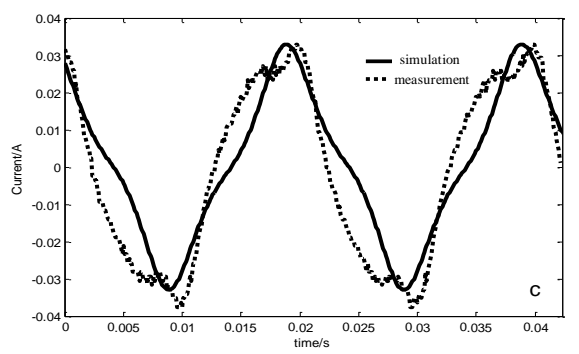

(c) c phase

Figure 8. A comparison of the exciting currents without DC bias

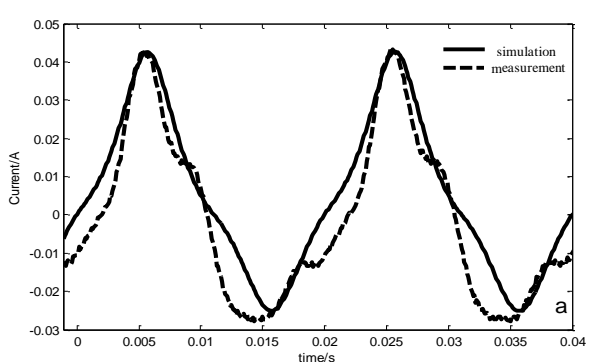

(a) a phase

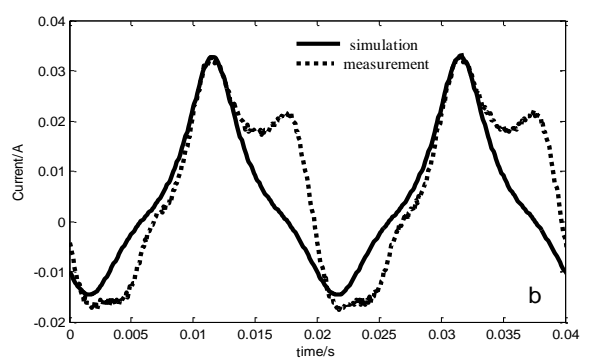

(b) b phase

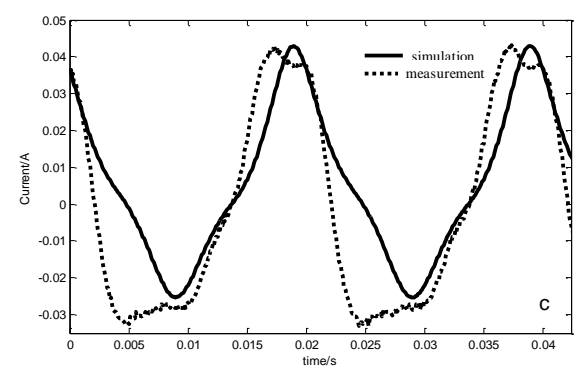

(c) c phase

Figure 9. A comparison of the exciting currents with DC bias

\section{CONCLUSIONS}

Each phase flux of the three-phase five-legged transformer interacts with each other. In the mathematical model, the iron core topology, the saturation characteristic of the core material and the influence of the eddy currents are considered sufficiently under DC bias. The exciting currents get distorted, and the nonlinear characteristic curves get saturation or deviation when the DC current invasion into the three-phase five-legged transformer. The simulation results and experiment results are compared. They are in good agreement.

\section{REFERENCES}

[1] M.A.S. Masoum, P.S. Moses, Impact of balanced and unbalanced direct current bias on harmonic distortion generated by asymmetric three--phase three--leg transformers[J]. IET Electr. Power Appl. 2010,4(7):507-515.

[2] Yang Yongming, Liu Xingmou, Chen Tao, et al. Impact of soil structure adjacent to ground electrodes of UHVDC power transmission lines on DC bias of power transformers[J]. Power System Technology, 2012, 36(7):26-32.

[3] Cui Mingde, Liu Chunming, Liu Lianguang. Assessment of the influence caused by solar storm on sichuan power grid rated 500 kV[J]. High Voltage Engineering, 2010, 36(11):2849-2855.

[4] Wang Jin-gang, Mao Kai, Duan $\mathrm{Xu}$, et al. Simulation and test of transformer vibration under DC bias[J]. Electric Machines and Control, 2015,19(1):58-67. 
[5] Zhao Zhigang, Liu Fuguil, Cheng Zhiguang, et al. Eddy current Loss of Copper Shielding Under DC--biased Condition in HVDC [J]. High Voltage Engineering. 2011,37(4):990-995.

[6] Guo Jie, Huang Hai, Tang Xin, et al. Analysis on $500 \mathrm{kV}$ Power Transformer Vibration Under DC M agnetic Biasing[J]. Power System Technology, 2012,36(3):70-75.

[7] Li Xiaoping, Wen Xishan. DC Bias Computation Study on Threephase Five Limbs Transformer[J]. Proceedings of the CSEE, 2010,30(1):127-131.

[8] Zhao Xiaojun, Zhang Xiaoxin, Li Huiqi, et al. Frequency Domain Coupled Model between Magnetic and Electric Circuits of DC Biased Transformers by Harmonic Balance Method[J]. Transactions of China Electrotechnical Society. 2014,29(9):211218.

[9] Zhao Xiaojun, Li Lin, Cheng Zhiguang. et a1. Analysis of the DC bias phenomenon in transformers based on harmonic-balanced finite element method[J]. Proceedings of the CSEE, 2010,30(21) : 103-108.

[10] Zhao Z, Liu F, Cheng Z. Measurements and calculation of core-based B-H curve and magnetizing current in DC-biased transformers[J], IEEE Transactions on Applied Super conductivity, 2010, 20(3):1131-1134.

[11] Zhu Yiying, Jiang Weiping, Zeng Zhaohua, et al. Studyiing on Measures of Restraining DC current Through Transformer Neutrals[J]. Proceedings of the CSEE,2005,25(13):1-7.

[12] Huang FuCheng, Ruan Jiangjun, Zhang Yu, et al. DC Magnetic Bias Induced Current Effects on Transformer and Restricting Methods[J]. High Voltage Engineering,2006,32(9):117-120.

[13] Lu Changbai, Zhu Yinghao. Theory and calculation of power transformer[M]. 2006: LiaoNing Science and Technology Press. 